\title{
Significance of chemical reaction on MHD near stagnation point flow towards a stretching sheet with radiation
}

\author{
S. Harinath Reddy ${ }^{1}$ K. Kumaraswamy Naidu ${ }^{2}$ D. Harish Babu ${ }^{2} \cdot$ P. V. Satya Narayana ${ }^{3}$ M. C. Raju ${ }^{4}$
}

Received: 31 March 2020 / Accepted: 3 October 2020 / Published online: 15 October 2020

(c) Springer Nature Switzerland AG 2020

\begin{abstract}
The influence of variable surface temperature and concentration on MHD stagnation point flow towards a stretching sheet plays an important role in wire coating, film blowing, fiber spinning and coating. In this work an attempt has been made to study the ramification of thermal radiation on MHD chemically reacting liquid past a stretching surface with nonlinear temperature and concentration. The nonlinear coupled governing equations are changed into a set of nonlinear ordinary differential equations by adopting similarity transformations. The set of nonlinear equations together with the boundary conditions are solved computationally by employing shooting technique. The influence of various flow field parameters (magnetic parameter, suction/injection parameter, velocity ratio parameter, heat absorption, radiation, chemical reaction parameter, Schmidt number) on momentum, heat measure, diffusion, skin friction coefficient, rate of heat transfer and rate of mass transfer is depicted and discussed in detail. The outcomes disclosed that the fluid temperature accelerates on rising the thermal radiation and reverse trend with heat absorption parameter. Species concentration diminishes on enhancing the chemical reaction parameter. A comparison has been made with the published results as a particular case and found to be in fair agreement.
\end{abstract}

Keywords Power law form of surface temperature and concentration $\cdot$ MHD $\cdot$ Thermal radiation $\cdot$ Chemical reaction

List of symbols

$B_{0}$

$a, b, c, n$

$c_{f x}$

$c_{p}$

$\tilde{C}_{w}(x)=\tilde{C}_{\infty}+c x^{n}$

$f^{\prime}$

$k$

$K r=\frac{K r_{1} \delta^{2}}{v}$

$K_{s}$

$R=\frac{4 \sigma^{*} \tilde{T}_{\infty}^{3}}{K_{s} k}$

$N u_{x}$

$M=\frac{\sigma B_{0}^{2}}{a \rho}$
Uniform magnetic field

Constants

Skin-friction coefficient

Specific heat at constant pressure

$\left(\mathrm{J} \mathrm{kg}^{-1} \mathrm{~K}\right)$

Concentration of the sheet

Dimensionless velocity

Thermal conductivity of fluid

$\left(\mathrm{W} \mathrm{m} \mathrm{m}^{-1} \mathrm{k}^{-1}\right.$ )

Chemical reaction parameter

Rosseland absorption coefficient

Radiation parameter

Nusselt number

Magnetic parameter

$\operatorname{Pr}=\frac{v}{\alpha}$
$q_{r}$
$q_{w}$
$\operatorname{Re} e_{x}$
$S h_{x}$
$\tilde{T}$
$\tilde{T}_{\infty}$

$\tilde{T}_{w}(x)=\tilde{T}_{\infty}+c x^{n}$

$\tilde{u}, \tilde{v}$

$\tilde{U}_{w}(x)=a x$

$\tilde{U}_{\infty}(x)=b x$

$\tilde{V}_{w}(x)=-(a v)^{0.5} S$

$S$
Prandtl number

Radiative heat flux $\left(\mathrm{W} \mathrm{m}^{-1}\right)$

Surface heat flux

Local Reynolds number

Sherwood number

Fluid temperature $(K)$

Temperature far away from the wall

(K)

Temperature of the sheet

Velocity components in $x$-, $y$-directions, respectively $\left(\mathrm{m} \mathrm{s}^{-1}\right)$

Stretching velocity

Free stream velocity

Mass flux velocity

$S>0$ suction and $S<0$ injection

D. Harish Babu, harish225babu@gmail.com | 'Department of Mathematics, Annamacharya Institute of Technology and Sciences, Rajampet, A.P., India. ${ }^{2}$ Department of Mathematics, Sree Vidyanikethan Engineering College, Tirupati, A.P. 517 102, India. ${ }^{3}$ Department of Mathematics, SAS, VIT, Vellore, T.N 632 014, India. ${ }^{4}$ Department of Mathematics, JNTUA College of Engineering, Pulivendula, A.P., India. 


\begin{tabular}{|c|c|}
\hline \multicolumn{2}{|l|}{ Greek symbols } \\
\hline$\alpha$ & The thermal diffusivity of the fluid \\
\hline$\gamma=\frac{Q}{a \rho c_{p}}$ & $\begin{array}{l}\text { Dimensionless heat generation/ } \\
\text { absorption coefficient }\end{array}$ \\
\hline$\eta$ & Similarity variable \\
\hline$\in=\frac{b}{a}$ & Velocity ratio parameter \\
\hline$\Omega=\frac{a}{\Omega k}$ & Permeability parameter \\
\hline$\mu$ & Dynamic viscosity \\
\hline$v$ & Kinematic viscosity $\left(\mathrm{m}^{2} \mathrm{~s}^{-1}\right)$ \\
\hline$\rho$ & Fluid density $\left(\mathrm{kg} \mathrm{m}^{-1}\right)$ \\
\hline$\sigma^{*}$ & 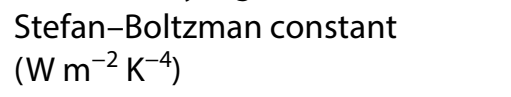 \\
\hline$\theta$ & Non-dimensional temperature \\
\hline$\psi$ & Stream function \\
\hline
\end{tabular}

Subscripts

$\begin{array}{ll}W & \text { Sheet surface } \\ \infty & \text { Infinity }\end{array}$

Superscript

Differentiation with respect to $\eta$

\section{Introduction}

Stagnation point flow induced by a variable heated stretching surface is frequently come across in many real-world technical applications such as: emergency core cooling systems, solar central receivers, blood flow problems, flow over tips of rockets, submarines and aircraft, glass production and crude oil purification. In view of these claims, Hayat et al. [1] explored the influence of radiation on MHD stagnation point flow past a vertical porous stretching plate. Pop et al. [2] investigated the influence of thermal radiation on the magneto-hydrodynamic stagnation point flow generated due to a sheet stretching. Bhattacharya et al. [3] presented the steady MHD stagnation point flow and heat transfer of a Casson fluid towards a stretching sheet. Bachok et al. [4] investigated the two-dimensional stagnation point flow over a stretching surface in the presence of nanofluid. Mukhopadhyay et al. [5] studied the combined effects of radiation and variable viscosity on the boundary layer stagnation point flow caused due to sheet stretching. Mabood et al. [6] discussed the simulation of the Cattaneo-Christov model near the stagnation point flow over a stretching surface. A few useful studies on MHD/ stagnation point flow over different geometrical aspects are discussed under refs. [7-13].

The impact of chemical reaction on the boundary layer flow has tremendous applications such as: in air and water emissions, fibrous insulation, air flows and many other chemical engineering problems. Devi et al. [14] inspected the influence of chemical reaction past a semi-infinite horizontal plate. Chamkha et al. [15] examined the chemical reaction and convection flow of the MHD micropolar fluid over a heated vertical plate. Satya Narayana et al. [16] discussed numerically the chemical reaction effect of non-Newtonian fluid over a stretching surface. Magyari and Chamka [17] examined the ramification of chemical reaction and heat absorption/generation on the micropolar liquid flow via a permeable stretching sheet. Ibrahim et al. [18] described the influence of induced magnetic field and chemical reaction on mass and heat transfer mixed convection flow past a vertical sheet. Hussain et al. [19] explored the significance of chemical reaction with ramped surface concentration over a moving plate. Sarojamma et al. [20] explored magnetohydrodynamic non-Newtonian Casson liquid flow past a stretching surface with chemical reaction under various conditions. Azhar et al. [21] deliberated the stagnation point flow of a Jeffrey fluid over a stretching surface with viscous dissipation. Recently, Yusuf et al. [22] demonstrated the effect of non-linear convection flow and chemical reaction in a channel with convective boundary conditions. Khan et al. [23] concentrated stagnation point flow of Buongiorno's nanofluid over radially stretching rotational disk. Numerous researchers [24-29] furnished the effect of chemical reaction/thermal radiation on different physical situations.

The literature reveals that the non-linear surface temperature and concentration play a pivotal role in many engineering and industrial applications such as polymer extrusion process. The research available in this direction is scanty. Hence this mathematical model aims to investigate the influence of chemical reaction and thermal radiation over stretching sheet by considering the power-law form of surface temperature and concentration. The constituted fluid flow equations are changed to ordinary coupled differential equations by utilizing suitable similarity transformations and are resolved by using a shooting technique in conjunction with Runge-Kutta scheme. The impact of prominent parameters on momentum, heat transfer, concentration, rate of heat and mass transfer are explored graphically and the outcomes of the numerical study are tabulated and briefly reported. Arrangement of the paper is as follows. The modeling of the problem with its constituted equations and boundary conditions are defined in Sect. 2 . The numerical approach is conferred in Sect. 3. The findings and discussion are represented in Sect. 4 and 5 respectively. Finally, conclusions are presented in Sect. 6. 


\section{Mathematical formulation of the problem}

A steady two dimensional incompressible, electrically conducting and chemically reacting MHD stagnation point flow generated due to a stretching sheet is considered. Let a homogeneous magnetic field is applied in the direction of $x$-axis which is normal to $y(>0)$-direction. The coordinate system and physical model of the problem are depicted in Fig. 1.

Based on aforesaid physical assumptions, the governing flow equations and corresponding conditions are represented by [30].

$\frac{\partial \tilde{u}}{\partial x}+\frac{\partial \tilde{v}}{\partial y}=0$

$\tilde{u} \frac{\partial \tilde{u}}{\partial x}=-\tilde{v} \frac{\partial \tilde{u}}{\partial y}+\tilde{U}_{\infty} \frac{\partial \tilde{U}}{\partial x}+\tilde{v} \frac{\partial^{2} \tilde{u}}{\partial y^{2}}-\frac{1}{\rho} B_{0}^{2} \sigma\left(\tilde{u}-\tilde{u}_{\infty}\right)-\frac{v}{K} \tilde{u}$

$\tilde{u} \frac{\partial \tilde{T}}{\partial x}+\tilde{v} \frac{\partial \tilde{T}}{\partial y}=\alpha \frac{\partial^{2} \tilde{T}}{\partial y^{2}}+\frac{1}{\rho} \frac{Q}{c_{p}}\left(\tilde{T}-\tilde{T}_{\infty}\right)-\frac{1}{\rho c_{p}} \frac{\partial q_{r}}{\partial y}$

$\tilde{u} \frac{\partial \tilde{C}}{\partial x}+\tilde{v} \frac{\partial \tilde{C}}{\partial y}=D \frac{\partial^{2} \tilde{C}}{\partial y^{2}}-K r_{1}\left(\tilde{C}_{\infty}-\tilde{C}\right)$

Subject to the boundaries

At $y=0$ :

$\tilde{u}=\tilde{U}_{w}(x), \tilde{v}=\tilde{V}_{w}(x), \tilde{T}=\tilde{T}_{w}(x), \tilde{C}=\tilde{C}_{w}(x)$

As $y \rightarrow \infty$ :

$\tilde{u} \rightarrow \tilde{U}_{\infty}(x), \tilde{T} \rightarrow \tilde{T}_{\infty}, \tilde{C} \rightarrow \tilde{C}_{\infty}$

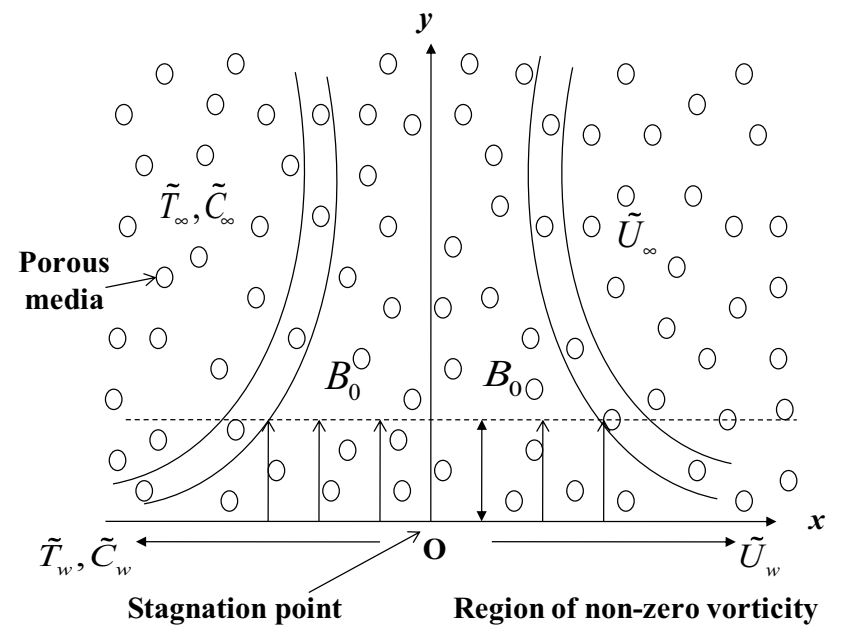

Fig. 1 Physical configuration
The radiative heat flux $q_{r}$ is defined as $q_{r}=-\frac{4}{3} \frac{\sigma^{*}}{k_{s}} \frac{\partial \tilde{T}^{* 4}}{\partial y}$ by utilizing the Rosseland approximation [31]. Use $-\frac{\partial q_{r}}{\partial y}=\frac{16}{3} \frac{\sigma^{*} T_{\text {o }}^{3}}{K_{s}} \frac{\partial^{2} \tilde{T}}{\partial y^{2}}$ such that eq. (3) becomes

$\tilde{u} \frac{\partial \tilde{T}}{\partial x}+\tilde{v} \frac{\partial \tilde{T}}{\partial y}=\alpha \frac{\partial^{2} T}{\partial y^{2}}+\frac{1}{\rho} \frac{Q}{c_{p}}\left(\tilde{T}-\tilde{T}_{\infty}\right)+\frac{16}{3 \rho} \frac{\sigma^{*} \tilde{T}_{\infty}^{3}}{k c_{p}} \frac{\partial^{2} \tilde{T}}{\partial y^{2}}$

where $\tilde{T}^{*^{4}}=4 \tilde{T} \tilde{T}_{\infty}^{3}-3 \tilde{T}_{\infty}^{4}$

Introducing subsequent similarity transformations [30]:

$\eta=y \cdot \sqrt{a / v^{\prime}} \theta(\eta)=\frac{\left(\tilde{T}_{\infty}-\tilde{T}\right)}{\left(\tilde{T}_{\infty}-\tilde{T}_{w}\right)}, \psi=x \sqrt{a v} f(\eta), \phi(\eta)=\frac{\left(\tilde{C}_{\infty}-\tilde{C}\right)}{\left(\tilde{C}_{\infty}-\tilde{C}_{w}\right)}$

In view of Eq. (8), the Eqs. (2)-(6) are transformed to:

$f^{\prime \prime \prime \prime}=-f f^{\prime \prime}+f^{\prime 2}+\epsilon^{2}-M\left(\in-f^{\prime}\right)+\Omega f^{\prime}$

$(1+4 R / 3) \theta^{\prime \prime}=-\operatorname{Pr}\left[(f) \theta^{\prime}-n f^{\prime} \theta+\gamma \theta\right]$

$\phi^{\prime \prime}=-S c\left(f \phi^{\prime}-n \phi f^{\prime}-K r \phi\right)$

$f^{\prime}(0)=s, f^{\prime}(\infty)=\in, f(0)=0, \theta(0)=1, \theta(\infty)=0, \phi(0)=1, \phi(\infty)=0$

Physical quantities are skin-friction $C_{f x}$, Nusselt number $\mathrm{Nu}_{x}$ and Sherwood number $\mathrm{Sh}_{x}$ (these are useful in numerous branches of engineering) represented as follows

$C_{f x}=\frac{\tau_{w}}{\rho u_{e}^{2}}=\operatorname{Re}_{x}^{-0.5} f^{\prime \prime}(0)$

$N u_{x}=\frac{1}{k} \frac{x \cdot q_{w}}{\left(T_{w}-T_{\infty}\right)}=-\operatorname{Re}_{x}^{0.5}\left(1+\frac{4}{3} R\right) \theta^{\prime}(0)$

$S h_{x}=\frac{x \cdot m_{w}}{D\left(C_{w}-C_{\infty}\right)}=-\operatorname{Re}_{x}^{0.5} \phi^{\prime}(0)$

\section{Numerical procedure}

The nonlinear boundary values problem (BVP) described by Eqs. (9)-(11) subject to the boundaries given in Eq. (12) is solved by employing numerical technique i.e. shooting method with R-K fourth order method [32]. In this method, the system of equations are reduced into first order ODE's by employing: $\alpha_{1}=f, \alpha_{2}=f^{\prime}, \alpha_{3}=f^{\prime \prime}$ ${ }_{,} \alpha_{4}=\theta, \alpha_{5}=\theta^{\prime}, \alpha_{6}=\phi, \alpha_{7}=\phi^{\prime}$ as follows 


$$
\left(\begin{array}{c}
\alpha_{1}^{\prime} \\
\alpha_{2}^{\prime} \\
\alpha_{3}^{\prime} \\
\alpha_{4}^{\prime} \\
\alpha_{5}^{\prime} \\
\alpha_{6}^{\prime} \\
\alpha_{7}^{\prime}
\end{array}\right)=\left(\begin{array}{c}
\alpha_{2} \\
\alpha_{3} \\
\alpha_{5} \\
\alpha_{2}^{2}+\Omega \alpha_{2}-\alpha_{1} \alpha_{3}-M\left(\in-\alpha_{2}\right)-\epsilon^{2} \\
\frac{-1}{1+\frac{4 R}{3}}\left[\operatorname{Pr}\left(\alpha_{1} \alpha_{5}-n \alpha_{2} \alpha_{4}+\gamma \alpha_{4}\right]\right. \\
\alpha_{7} \\
\operatorname{Sc}\left[n \alpha_{6} \alpha_{2}-\alpha_{1} \alpha_{7}+k_{r} \alpha_{6}\right]
\end{array}\right) \text { with }\left(\begin{array}{c}
\alpha_{1} \\
\alpha_{2} \\
\alpha_{3} \\
\alpha_{4} \\
\alpha_{5} \\
\alpha_{6} \\
\alpha_{7}
\end{array}\right)=\left(\begin{array}{c}
S \\
1 \\
x_{1} \\
0 \\
x_{2} \\
0 \\
x_{3}
\end{array}\right)
$$

The above system of equations are solved computationally with the help of shooting technique depending on the $\mathrm{R}-\mathrm{K}$ fourth order method by choosing the suitable missing conditions $\alpha_{3}, \alpha_{5}$ and $\alpha_{7}$. However, the step size and convergence criteria are fixed at 0.001 and $10^{-6}$ respectively.

\section{Results}

Influence of chemical reaction and radiation on MHD stagnation point flow over a stretching surface with heat source is examined numerically. This section presents the influence of different physical parameters on velocity, heat measure, concentration, skin friction, rate of heat transfer, Sherwood number through Figs. 2, 3, 4, 5, 6, 7, 8, 9, 10, 11 and 12 .

The velocity profile is described respectively in Figs. 2 and 3 for different values of injection and suction parameters. It is noticed that the velocity of fluid increases with decreasing the injection parameter $(S<0)$, while opposite behavior is seen with increasing suction parameter $(S>0)$.

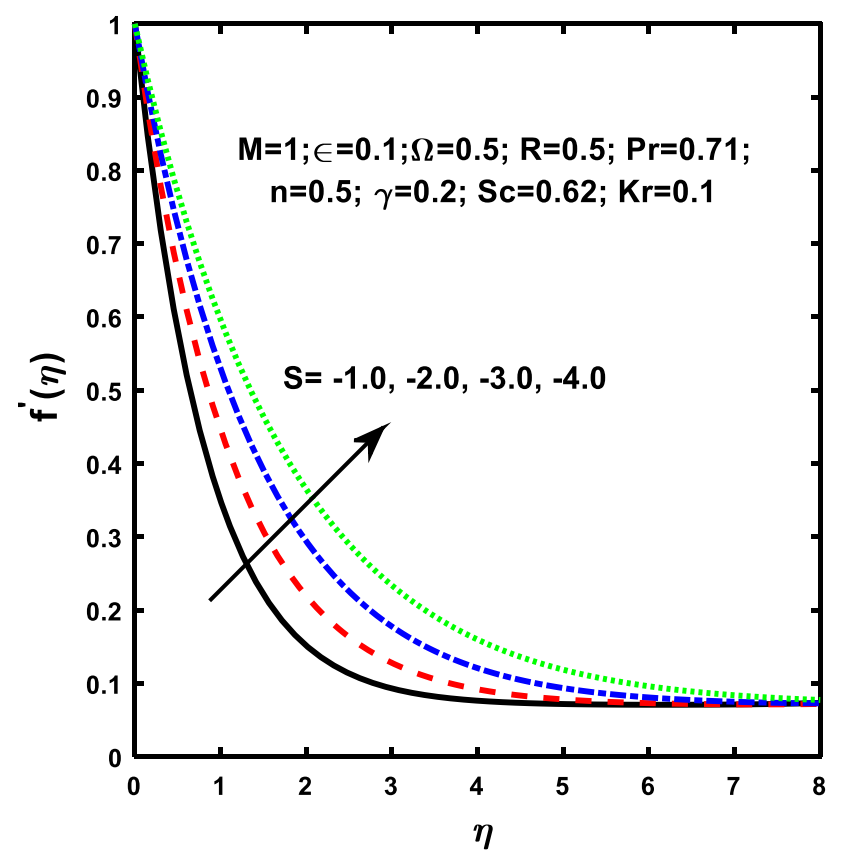

Fig. 2 Variation of Injection parameter $S(<0)$ on $f^{\prime}(\eta)$

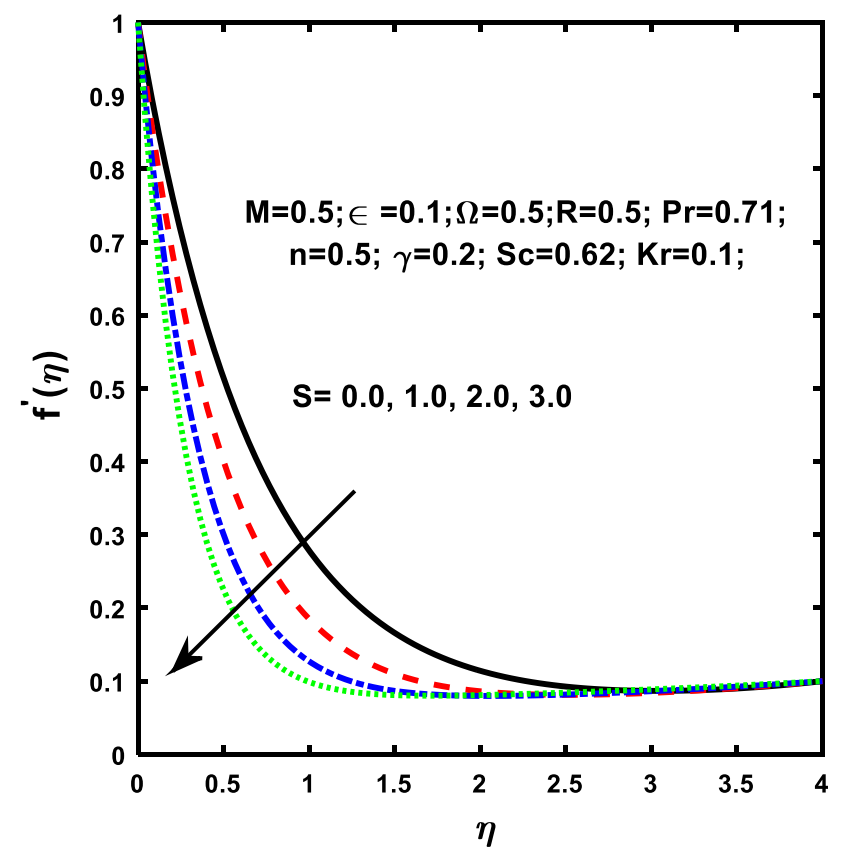

Fig. 3 Variation of Suction parameter $S(\geq 0)$ on $f^{\prime}(\eta)$

Figure 4 portrayed that the velocity profile for different values of $M$. From this figure, it can be seen that the velocity profile decays on enhancing the values of $M$. Owing to the reality that, the application of the transverse magnetic field appears to give rise to a resistive kind of force known as Lorentz force which impedes the velocity profile. Thus, the impact of $M$ leads to diminish the velocity field.

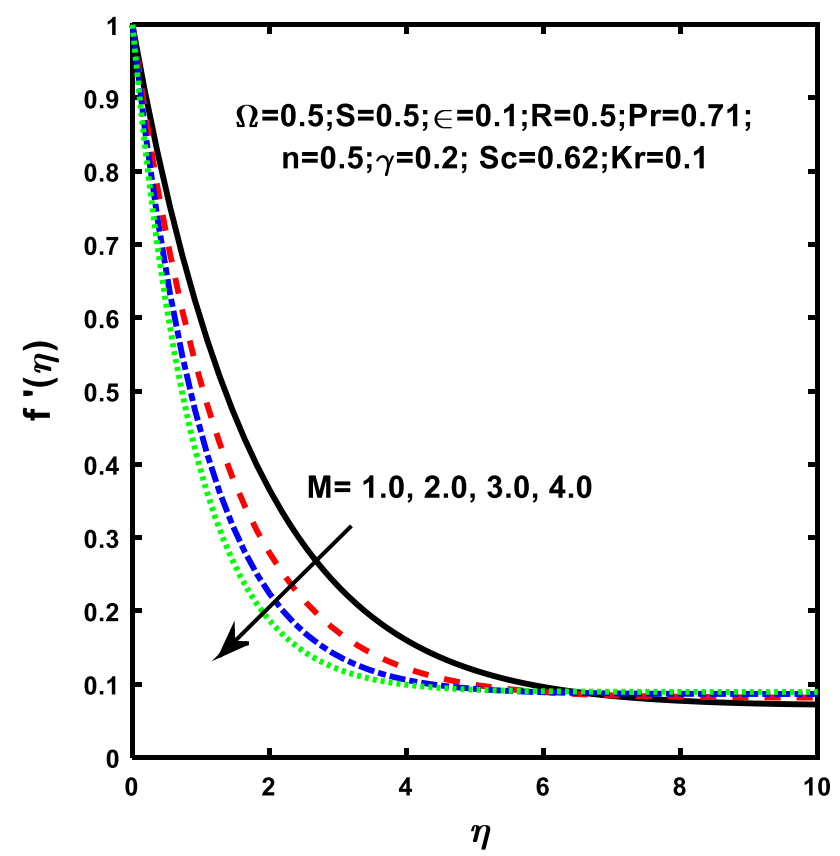

Fig. 4 Variation of magnetic field $M$ on $f^{\prime}(\eta)$ 


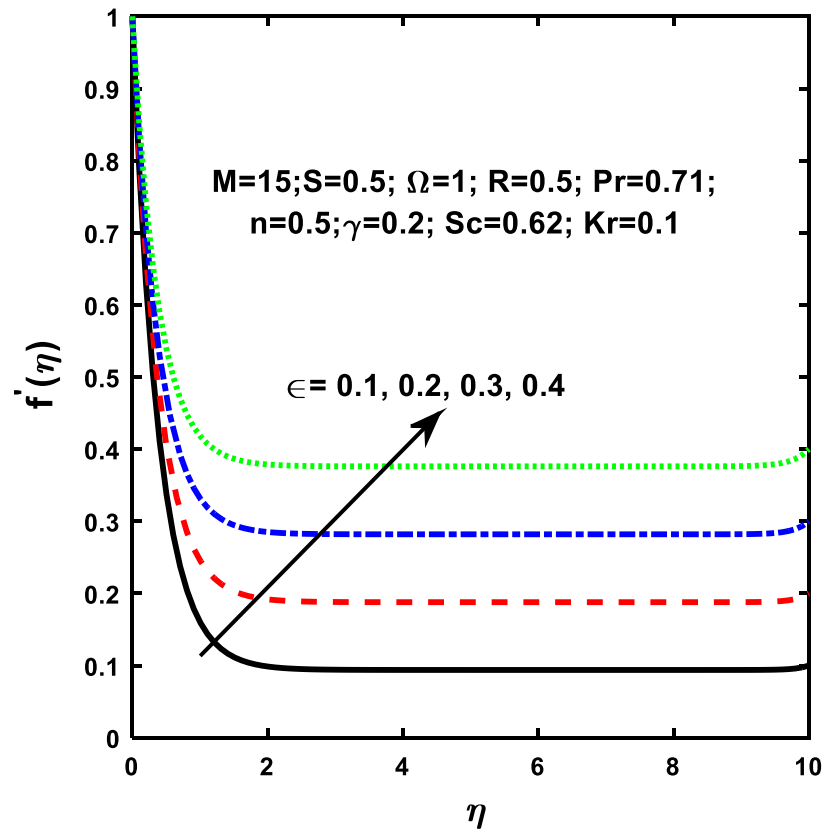

Fig. 5 Variation of ratio parameter $\in$ on $f^{\prime}(\eta)$

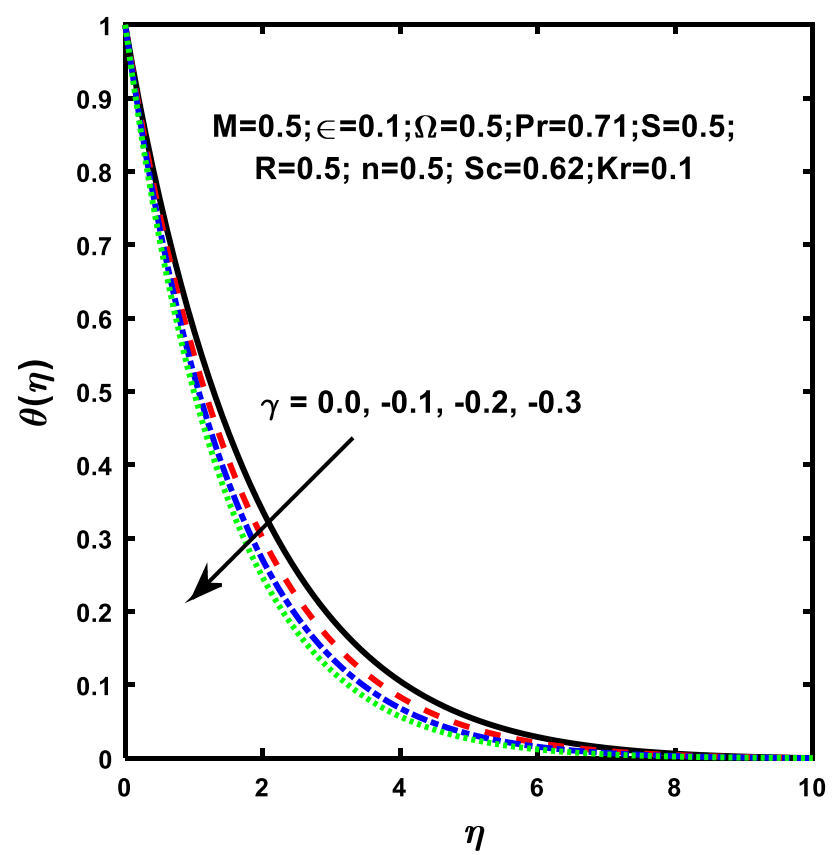

Fig. 6 Variation heat absorption parameter $\gamma$ on $\theta(\eta)$

Figure 5 depicts the effect of velocity ratio parameter $\epsilon$ on $f^{\prime}(\eta)$. It is seen that $f^{\prime}(\eta)$ boosts on enhancing the ratio parameter $\in$.

Figures 6 and 7 illustrate the variation of temperature for the distinct values of heat absorption parameter $\gamma$ and the radiation parameter $R$. From Fig. 6 it is observed that the non-dimensional temperature declined with an

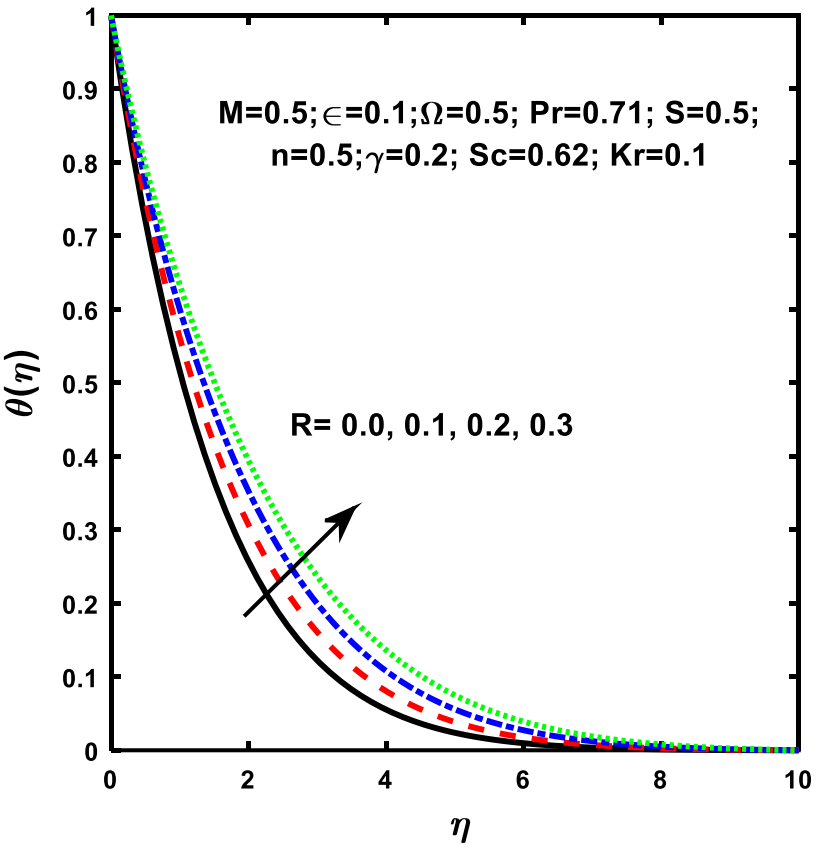

Fig. 7 Variation of Radiation parameter $R$ on $\theta(\eta)$

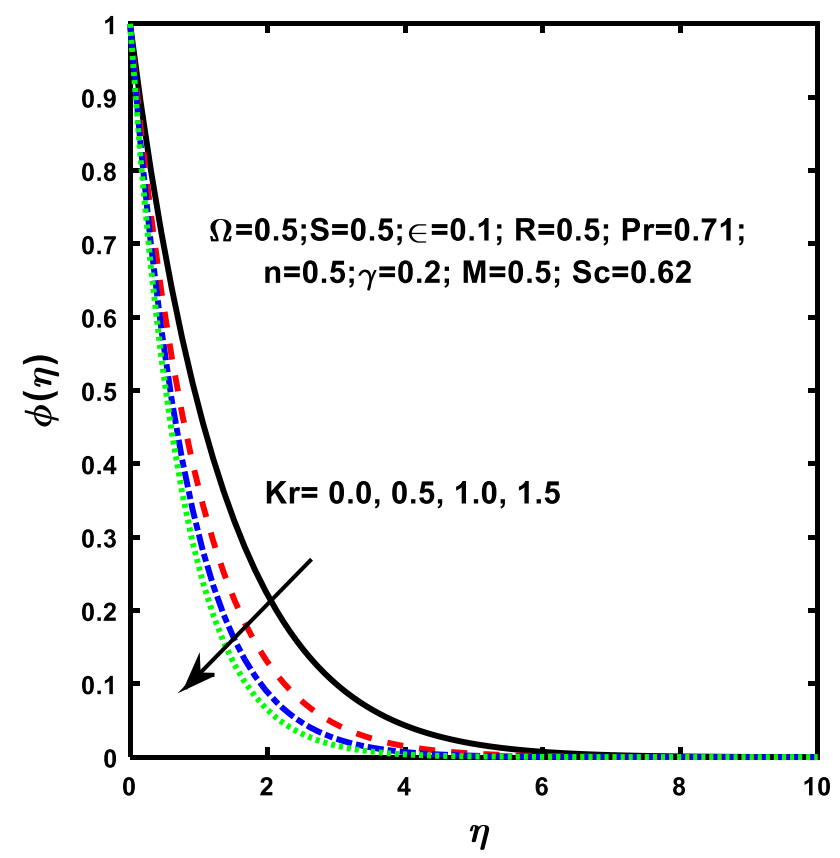

Fig. 8 Variation of Chemical reaction parameter $\operatorname{Kr}$ on $\phi(\eta)$

increase in $\gamma$. Physically, the temperature falls when the intensity of heat is minimized. From Fig. 7 it is perceived that the fluid temperature augments on rising values of $R$. This is because the higher thermal radiation value means a greater heat flux that increases the fluid temperature.

The impact of the chemical reaction parameter $\mathrm{Kr}$ and the Schmidt number $S c$ on the species concentration is 


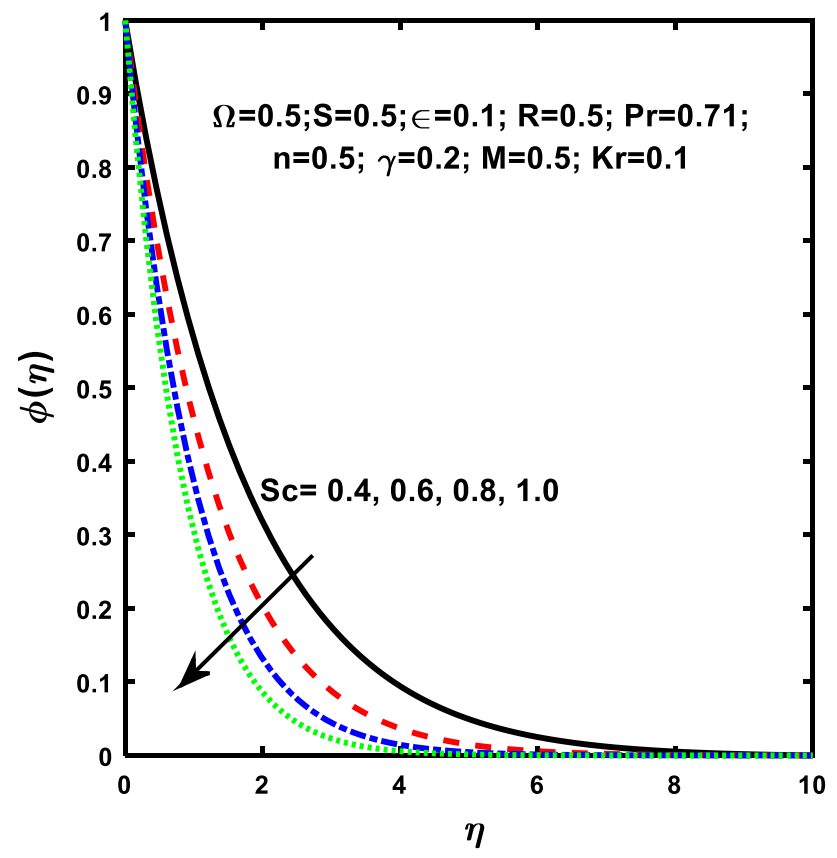

Fig. 9 Variation of Schmidt number Sc on $\phi(\eta)$
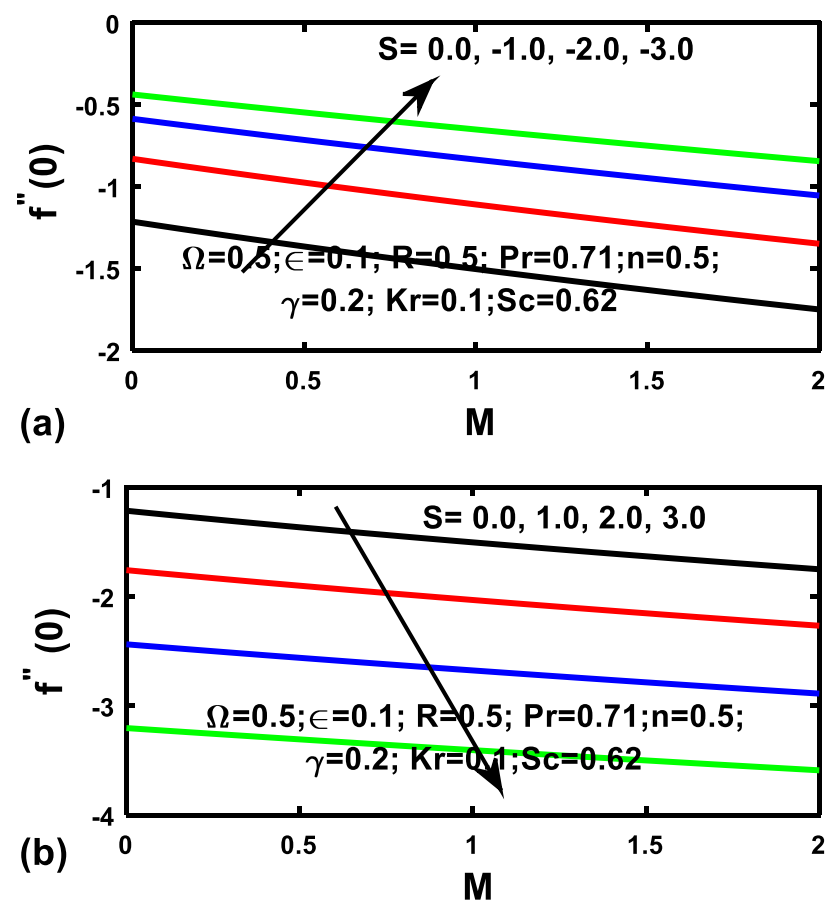

Fig. 10 a, b Variation of $S$ on Skin friction coefficient against $M$

displayed respectively in Figs. 8 and 9. The concentration profile decelerates on rising $K r$. It is true because the higher values of chemical reaction make fall in the chemical molecular diffusivity. The concentration profile retards on enhancing $S c$ as noticed in Fig. 9. This is due to the fact that the molecular diffusivity decreases with increase in

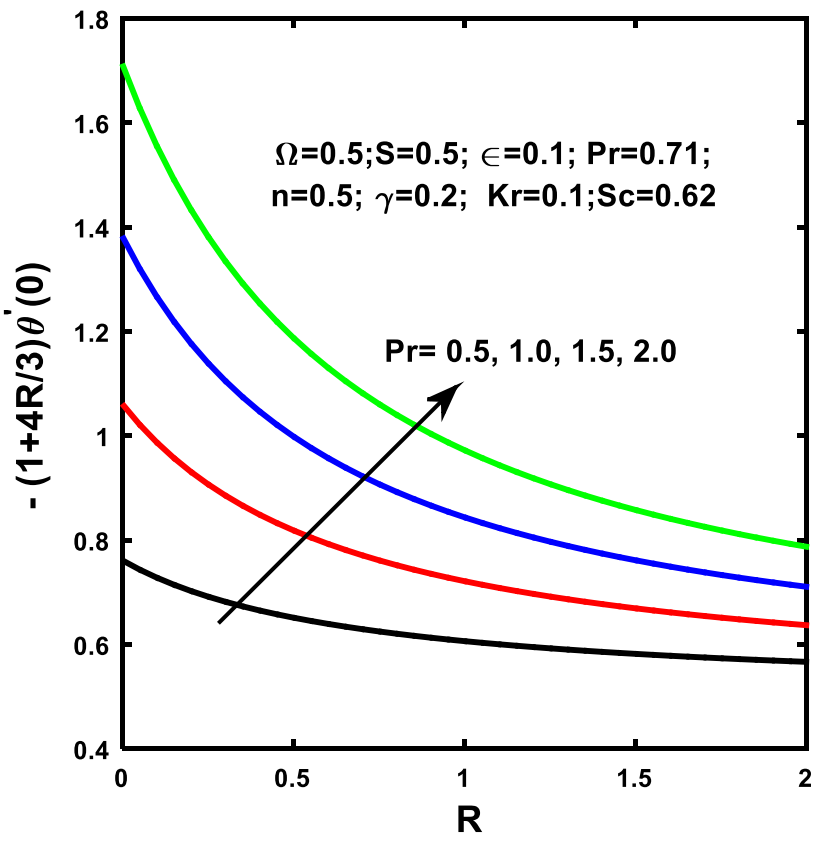

Fig. 11 Variation of Pron Nusselt number against $R$

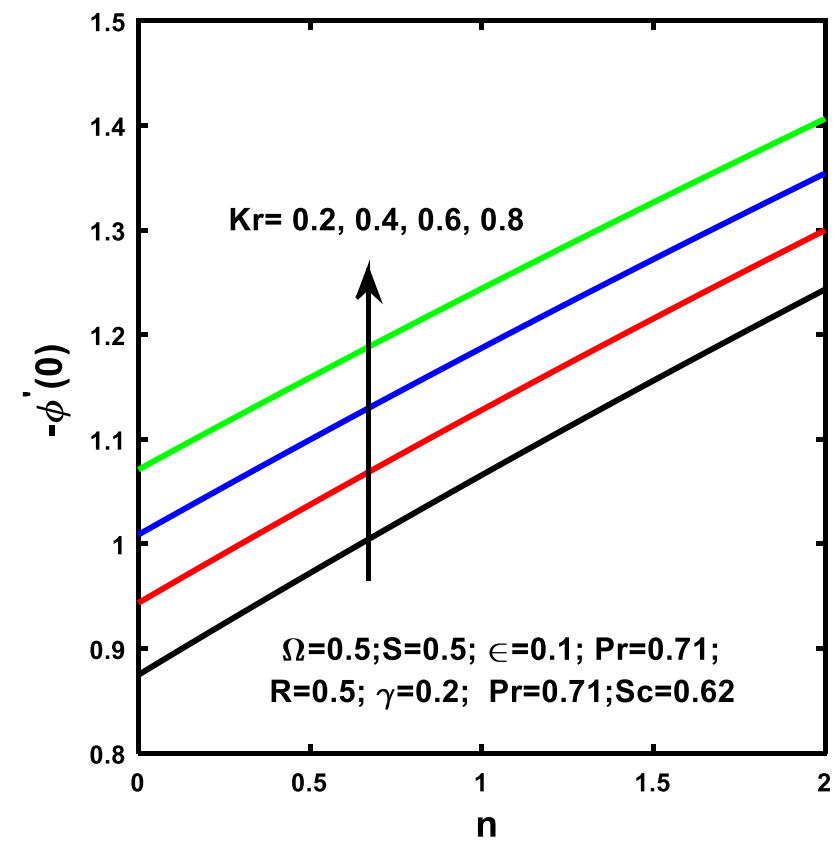

Fig. 12 Variation of $K r$ on Sherwood number against $n$

Schmidt number. Therefore, the species concentration is high for smaller values of $S c$ and low for superior values of Sc. Figure $10 a, b$ represent the effect of skin-friction coefficient $C f_{x}$ against the magnetic parameter $M$. It is obvious that $C f_{x}$ decrease for decreasing values of $S$ which is shown in Fig. 10a and the reverse trend is noticed for increasing values of $S$ in Fig. 10b. Also noticed that $C f_{x}$ decreases with 
the rise of magnetic parameter $M$ for both suction and injection. The variation of $-\left(1+\frac{4}{3} R\right) \theta^{\prime}(0)$ for different values of Prandtl number $\operatorname{Pr}$ and radiation parameter $R$ is shown in Fig. 11. It is observed that $-\left(1+\frac{4}{3} R\right) \theta^{\prime}(0)$ augments with an increase in $P r$ and the reverse trend has seen on augmenting the radiation parameter $R$. The effect of $K r$ and $n$ on $-\phi^{\prime}(0)$ is portrayed in Fig. 12. It is evident that $-\phi^{\prime}(0)$ increases with an increase in the chemical reaction parameter $K r$ as well as $n$.

\section{Discussion}

The influence of variable surface temperature $T_{w}(x)=T_{\infty}+A x^{r}$ on laminar flow due to a sheet stretching was theoretically presented by Xu et al. [33]. Further heat and mass transfer of non-Newtonian nanofluid nearby stagnation point flow impinge an oblique wall under the constant condition as $T^{*}=T_{w}, C^{*}=C_{w}$ was studied by Nadeem et al. [34]. Also, Pal et al. [35] studied the impact of viscous scattering and radiation of the flow nearby stagnation point over the nanofluid stretching surface under the conditions $u_{w}= \pm c x, T_{w}-T_{\infty}=b\left(\frac{1}{x}\right)^{-2}$. More recently, Fenuga et al. [36] demonstrated the heat transfer rate on the non-linear vertical sheet near the stagnation point. In view of these, the present work is focused on the significance of chemical reaction and thermal radiation nearby stagnation point by considering the power-law form of surface temperature $\tilde{T}_{w}(x)=\tilde{T}_{\infty}+c x^{n}$ and surface concentration $\tilde{C}_{w}(x)=\tilde{C}_{\infty}+c x^{n}$. The governing equations are solved numerically by using RK method scheme along with shooting technique. Further, the characteristics of skin-friction coefficient, rate of heat transfer are discussed in Tables 1 and 2 for pertinent physical parameters.

In Table 1, a comparison has made for $f^{\prime \prime}(0)$ when $M=S=\Omega=R=K r=S C=0$ with Ibrahim et al. [37], Hayat et al. [38] and Agbaje et al. [30] by giving different values to $\in$. It is found that $f^{\prime \prime}(0)$ increases with an increase in the velocity ratio parameter. Table 2 presents the
Table 2 A numerical analogy for $-\left(1+\frac{4}{3} R\right) \theta^{\prime}(0)$ contrary those of Ibrahim et al. [37] and Agbaje et al. [30] for diverse values of $\in$ and $\operatorname{Pr}$, when $M=S=\Omega=R=K r=S C=n=\gamma=0$

\begin{tabular}{llllll}
\hline$\in$ & Pr & Ibrahim et al. [37] & \multicolumn{2}{c}{ Agbaje et al. [30] } & Present \\
\cline { 4 - 5 } & & & SPM & SQLM & \\
\hline 0.1 & 1.0 & 0.6022 & 0.6022 & 0.6022 & 0.602213 \\
0.2 & 1.0 & 0.6245 & 0.6245 & 0.6245 & 0.624656 \\
0.5 & 1.0 & 0.6924 & 0.6924 & 0.6924 & 0.692455 \\
0.1 & 1.5 & 0.7768 & 0.7768 & 0.7768 & 0.776815 \\
0.2 & 1.5 & 0.7971 & 0.7971 & 0.7971 & 0.797129 \\
0.5 & 1.5 & 0.8648 & 0.8648 & 0.8648 & 0.864800 \\
\hline
\end{tabular}

comparison of $\quad-\left(1+\frac{4}{3} R\right) \theta^{\prime}(0) \quad$ for $M=S=\Omega=R=K r=S C=n=\gamma=0$ with Ibrahim et al. [37] and Agbaje et al. [30] for different values of $\operatorname{Pr}$ and $\in$. It is observed that Nusselt number enhances on augmenting Pr and $\in$. From these tables, it is thus apparent that the current results are in very good agreement.

\section{Conclusion}

In this article, the significance of radiation and chemical reaction on MHD stagnation point flow due to sheet stretching is investigated. Power law form of surface temperature and concentrations are considered in this mathematical model. The flow governing equations are solved computationally. The key results in the current research are listed as follows:

1. Fluid momentum amplifies on raising the velocity ratio parameter and opposite trend with suction and magnetic parameters.

2. The dimensionless fluid temperature accelerates for augmenting the thermal radiation parameter and retards with heat absorption parameter.

3. Species concentration retards for rising values of chemical reaction parameter and Schmidt number.
Table 1 A numerical analogy for $f^{\prime \prime}(0)$ contrary those of Ibrahim et al. [37], Hayat et al. [38] and Agbaje et al. [30] for diverse values of $\in$, when $M=S=\Omega=R=K r=S c=0$

\begin{tabular}{|c|c|c|c|c|c|}
\hline \multirow[t]{2}{*}{$\in$} & \multirow[t]{2}{*}{ Ibrahim et al. [37] } & \multirow[t]{2}{*}{ Hayat et al. [38] } & \multicolumn{2}{|c|}{ Agbaje et al. [30] } & \multirow[t]{2}{*}{ Present } \\
\hline & & & SPM & SQLM & \\
\hline 0.01 & -0.9980 & -0.99802 & -0.9980 & -0.9980 & -0.998043 \\
\hline 0.02 & - & -0.99578 & -0.9958 & -0.9958 & -0.996083 \\
\hline 0.05 & - & -0.98757 & -0.9876 & -0.9876 & -0.987729 \\
\hline 0.10 & -0.9694 & -0.96938 & -0.9694 & -0.9694 & -0.969436 \\
\hline 0.20 & -0.9181 & -0.91810 & -0.9181 & -0.9181 & -0.918112 \\
\hline 0.50 & -0.6673 & -0.66732 & -0.6673 & -0.6673 & -0.667260 \\
\hline 1.00 & - & 0.00000 & - & 0.0000 & 0.000000 \\
\hline
\end{tabular}


4. Skin friction-coefficient rises with suction parameter.

5. Nusselt number increases with growing Prandtl number.

The results of this model are useful in the fields of oil recovery, mass transfer, drying processes and solar Collectors, where variable surface heat and mass transfers are involved.

\section{Compliance with ethical standards}

Conflict of interest The authors declare that they have no conflict of interest.

\section{References}

1. Hayat T, Abbas Z, Pop I, Asghar S (2010) Effects of radiation and magnetic field on the mixed convection stagnation-point flow over a vertical stretching sheet in a porous medium. IntJ Heat Mass Transf 53:466-474

2. Pop I, Anuar I, Fazlina A (2011) Radiation effects on the MHD flow near the stagnation point of a stretching sheet: revisited. Zeitschrift Fur AngewandteMathematik Und Physik 62:953-956

3. Bhattacharyya K (2013) MHD stagnation-point flow of casson fluid and heat transfer over a stretching sheet with thermal radiation. J Thermodyn 2013:1-9

4. Bachok N, Ishak A, Pop I (2011) Stagnation-point flow over a stretching/shrinking sheet in a nanofluid. Nanoscale Res Lett 6:623

5. Mukhopadhyay S (2013) Effects of thermal radiation and variable fluid viscosity on stagnation point flow past a porous stretching sheet. Meccanica 48:1717-1730

6. Mabood F, Imtiaz M, Hayat T (2020) Features of Cattaneo-Christov heat flux model for Stagnation point flow of a Jeffrey fluid impinging over a stretching sheet: a numerical study. Heat Transf 49(5):1-11

7. Khashi'ie NS, Arifin NM, Rashidi MM, Ezad HH, Wahi N (2020) Magnetohydrodynamics (MHD) stagnation point flow past a shrinking/stretching surface with double stratification effect in a porous medium. J Therm Anal Calorim 139:3635-3648

8. Takhar HS, Chamkha AJ, Nath G (2000) MHD flow over a moving plate in a rotating fluid with magnetic field, hall currents and free stream velocity. Int J Eng Sci 38:1303-1314

9. Hafeez A, Khan M, Ahmed J (2020) Stagnation point flow of radiative Oldroyd-B nanofluid over a rotating disk. Comput Methods Progr Biomed 191:1-26

10. Chamkha AJ, Al-Mudhaf A (2005) Unsteady heat and mass transfer from a rotating vertical cone with a magnetic field and heat generation or absorption effects. Int J Therm Sci 44(3):267-276

11. Khedr MEM, Chamkha AJ, Bayomi M (2009) MHD flow of a micropolar fluid past a stretched permeable surface with heat generation or absorption. Nonlinear Anal Modell Control 14(1):27-40

12. Raza J (2019) Thermal radiation and slip effects on magnetohydrodynamic (MHD) stagnation point flow of Casson fluid over a convective stretching sheet. Propuls Power Res 8(2):138-146

13. Reddy PS, Chamkha AJ (2016) Soret and Dufour effects on MHD convective flow of $\mathrm{Al}_{2} \mathrm{O}_{3}$-water and $\mathrm{TiO} 2$-water nanofluids past a stretching sheet in porous media with heat generation/ absorption. Adv Powder Technol 27(4):1207-1218
14. Devi ASP, Kandasamy R (1999) Effects of chemical reaction, heat and mass transfer on laminar flow along a semi-infinite horizontal plate. Heat Mass Transf 35:465-467

15. Chamkha AJ, Mohamed RA, Ahmed SE (2010) Unsteady MHD natural convection from a heated vertical porous plate in a micropolar fluid with Joule heating, chemical reaction and radiation effects. Meccanica 46(2):399-411

16. Satya Narayana PV, Harish Babu D (2016) Numerical study of MHD heat and mass transfer of a Jeffrey fluid over a stretching sheet with chemical reaction and thermal radiation. J Taiwan Inst Chem Eng 59:18-25

17. Magyari E, Chamkha AJ (2010) Combined effect of heat generation or absorption and first-order chemical reaction on micropolar fluid flows over a uniformly stretched permeable surface: the full analytical solution. Int J Therm Sci 49(9):1821-1828

18. Ibrahim SM, Mabood F, Suneetha K, Lorenzini G (2017) Effect of chemical reaction on combined heat and mass transfer by laminar mixed convection flow from vertical surface with induced magnetic field and radiation. J Eng Thermophys 26(2):256-271

19. Hussain SM, Jain J, Seth GS, Rashidi MM (2017) Free convective heat transfer with hall effects, heat absorption and chemical reaction over an accelerated moving plate in a rotating system. J Magn Magn Mater 422:112-123

20. Sarojamma G, Sreelakshmi K, Animasaun IL (2019) Numerical study of non-linear thermal radiative heat transfer in a nonDarcy chemically reactive Casson fluid flow. SN Appl Sci 1:1136

21. Azhar E, Iqbal Z, Maraj EN (2019) Viscous dissipation performance on stagnation point flow of Jeffrey fluid inspired by internal heat generation and chemical reaction. Thermal Sci Eng Progress 13(1-6):100377

22. Yusuf TA, Mabood F, Gbadeyan JA, Adesanya SO (2020) Nonlinear convective flow for MHD Oldroyd 8-constant fluid in a channel with chemical reaction and convective boundary condition. J Thermal Sci Eng Appl 12(5):1-13

23. Khan M, El Shafey AM, Salahuddin T, Khan F (2020) Chemically Homann stagnation point flow of Carreau fluid. Phys $A$ 551:124066. https://doi.org/10.1016/j.physa.2019.124066

24. Mabood F, Ibrahim SM, Lorenzini (2017) Chemical reaction effects on MHD rotating fluid over a vertical plate embedded in porous medium with heat Source. J Eng Thermophys 26(3):399-415

25. Chamkha AJ (2001) Coupled heat and mass transfer by natural convection about a truncated cone in the presence of magnetic field and radiation effects. Numer Heat Transf A Appl 39(5):511-530

26. Harish Babu D, Samantha Kumari S, Satyanarayana PV (2020) Chemical reaction and thermophoresis effects on MHD mixed convection flow over an inclined porous plate with variable suction. In: Advances in fluid dynamics (LNME), pp 723-735

27. Sudarsana Reddy P, Sreedevi P, Chamkha AJ (2017) MHD boundary layer flow, heat and mass transfer analysis over a rotating disk through porous medium saturated by $\mathrm{Cu}$-water and Ag-water nanofluid with chemical reaction. Powder Technol 307:46-55

28. Makinde OD, Mabood F, Ibrahim SM (2018) Chemically reacting on MHD boundary layer flow of nanofluids over a nonlinear stretching sheet with heat source/sink and thermal radiation. Thermal Sci 22(1B):495-506

29. Chamkha AJ, Takhar HS, Soundalgekar VM (2001) Radiation effects on free convection flow past a semi-infinite vertical plate with mass transfer. Chem Eng J 84(3):335-342

30. Agbaje TM, Mondal S, Makukula ZG, Motsa SS, Sibanda P (2018) A new numerical approach to MHD stagnation point flow and heat transfer towards a stretching sheet. Ain Shams Eng J 9:233-243 
31. Rosseland S (1931) Astrophysik auf Atom-Theoretischer Grundlage. Springer, Berlin, pp 41-44

32. Na TY (1979) Computational method in engineering boundary value problems. Academic Press, New York

33. Xu H, Liao S (2009) Laminar flow and heat transfer in the boundary-layer of non-Newtonian fluids over a stretching flat sheet. Comput Math Appl 57(9):1425-1431

34. Nadeem S, Rashid M, Akbar NS (2013) Non-orthogonal stagnation point flow of a nano non-Newtonian fluid towards a stretching surface with heat transfer. Int J Heat Mass Transf 57(2):679-689

35. Pal D, Mandal G, Vajravelu K (2014) Flow and heat transfer of nanofluids at a stagnation point flow over a stretching/shrinking surface in a porous medium with thermal radiation. Appl Math Comput 238(1):208-224

36. Fenuga OJ, Hassan AR, Olanrewaju PO (2020) Mixed convection in MHD flow and heat transfer rate near a stagnation-point on a non-linear vertical stretching sheet. Int J Appl Mech Eng 25(1):37-51

37. Ibrahim W, Shankar B, Naneppanavar MM (2013) MHD stagnation point flow heat transfer due to nanofluid towards a stretching sheet. Int J Heat Mass Transf 56:1-9

38. Hayat T, Javed T, Abbas Z (2009) MHD flow of micropolar fluid near stagnation point towards non-linear stretching surface. Nonlinear Anal Real world Appl 10(3):1514-1526

Publisher's Note Springer Nature remains neutral with regard to jurisdictional claims in published maps and institutional affiliations. 